Benedikt George*, Markus Lehner, Michael Fink, Stefan J. Rupitsch, Pia T. Hiltl, Ula Savšek, Geoffrey Lee, and Helmut Ermert

\title{
Determination of the Cavitation Pressure Threshold in Focused Ultrasound Wave Fields applied to Sonosensitive, Biocompatible Nanoparticles for Drug Delivery Applications
}

https://doi.org/10.1515/cdbme-2020-3138

\begin{abstract}
Employing sonosensitive nanoparticles as carriers of active pharmaceutical ingredients emerges in ultrasonic Drug Delivery. Drug release can be initiated by focused ultrasound via the effect of inertial cavitation in certain target areas of particle loaded tissue. For stimulating inertial cavitation, a specific peak rarefaction pressure threshold must be exceeded. This pressure threshold has to be determined in order to estimate the risk of tissue damage during the drug release procedure. Therefore, this study provides a method to reliably verify the cavitation pressure threshold of sonosensitive and biocompatible nanoparticles.
\end{abstract}

Keywords: Drug Delivery, FUS, Cavitation Pressure Threshold, Nanoparticles

\section{Introduction}

Still one of the most devastating diseases in the world is cancer, pushing researchers to develop a successful treatment method for the cure of cancer patients. However, over the past years, mortality has decreased due to improved treatment modalities like radiation therapy, surgical interventions as well as chemotherapy to contain tumor growth. Though, these current treatment methods also impair healthy cell tissue and lead to considerable side effects. Especially, the systemically administered chemotherapeutic drug has an toxic effect on the entire body of the patient, which should be reduced.

A suitable approach to minimize toxic side effects is Drug Targeting, which is characterized by a selective accumulation of active pharmaceutical ingredient (API) to increase the con-

*Corresponding author: Benedikt George, Department of Sensor Technology, Paul-Gordan-Street 3/5, Erlangen, Germany, e-mail: benedikt.george@fau.de

Markus Lehner, Michael Fink, Stefan J. Rupitsch, Helmut Ermert, Department of Sensor Technology, Paul-Gordan-Street 3/5, Erlangen, Germany

Pia T. Hiltl, Ula Savšek, Geoffrey Lee, Department of Pharmaceutics, Cauerstraße 4, Erlangen, Germany centration of API in the tumorous tissue, while its concentration in healthy tissue is below a certain level to prevent toxic effects. Additionally, this localized drug delivery enables a significant reduction of the total amount of administered API.

In Drug Targeting (DT), various methods are applied to accumulate the drug into the tumorous tissue. These methods are based on physical principles as well as on physiological features of the tumorous tissue, e.g., Passive Targeting, exploiting abnormalities like the leaky vasculature of cell membrane to accumulate APIs passively, also known as the Enhanced Permeability and Retention Effect (EPR) [1, 2], or Thermal Targeting in which hyperthermia is commonly used as stimulus for triggered release of liposomal APIs [3, 4], or Magnetic Drug Targeting, which employs superparamagnetic iron oxide nanoparticles (SPIONs) as vehicles to guide the API to the tumor controlled by an external magnetic field $[5,6]$.

An emerging non-invasive treatment method is Ultrasonic Drug Targeting (UDT). This method is characterized by the fact that the release of the API from their sonosensitive carriers can be controlled by targeted sonication with focused ultrasound (FUS) via the effect of inertial cavitation (IC) [7, 8]. In this process - as mentioned at the DT methods above - the carrier particles required to transport the API close to the tumor, vary on their material and size very much.

In this contribution we focus on newly designed sonosensitive and biocompatible poly-L-lactid acid (PLA) formulations (spheres and capsules), which are planned to serve as drug carriers for drug delivery applications. Drug release is initiated via IC generated by a FUS transducer and is further enhanced due to the sonosensitive behavior of the carrier formulation.

As the release of the active ingredient is significantly influenced by FUS, a suitable transducer's excitation signal with corresponding parameters such as amplitude and frequency must be developed. With respect to clinical application, the signal development is quite challenging, since it is known that tissue can be irreparably damaged by FUS due to large peak rarefaction pressures (PRFP) and high US intensities. For a safe application of diagnostic ultrasound, limit values have 
been recommended by the FDA. The maximum applicable PRFP is set by the Mechanical Index $(M I)$, which is defined as the spatial-peak value $\hat{p}$ of the PRFP in MPa, derated by $0.3 \mathrm{~dB} / \mathrm{MHz} \mathrm{cm}$ at each point along the beam axis, divided by the square root of the ultrasound frequency $f$ in $\mathrm{MHz}$ [9]

$$
M I=\frac{\hat{p}}{\mathrm{MPa}} / \sqrt{\frac{f}{\mathrm{MHz}}} .
$$

For our clinical application, the release of the API should be instigated in the blood vessels leading to the tumor. Hence, we establish an $M I$ of 1.9 for peripheral vessels complying with FDA regulations for diagnostic ultrasound (Tab. 3 in [9]). In addition to the $M I$, the spatial-peak pulse-average intensity $I_{\text {SPPA.3 }}$ is mentioned, which is defined as the pulse-average intensity at the point in the acoustic field, where the pulseaverage intensity is a maximum or is a local maximum within a specified region, derated by $0.3 \mathrm{~dB} / \mathrm{MHz} \mathrm{cm}$ to account for the acoustic attenuation in soft tissues [9]. For peripheral vessels, the FDA sets the $I_{\mathrm{SPPA} .3}$ to $190 \mathrm{~W} / \mathrm{cm}^{2}$.

Under consideration of these limit values, we stimulate IC at frequencies $(650 \mathrm{kHz}-850 \mathrm{kHz})$ permitting sufficient focusing to the desired tissue region. Higher ultrasound frequencies in the MHz-range can be focused even better, but it is known that the cavitation pressure threshold (CPT) is notoriously high. Besides, stronger focusing of an ultrasound wave concentrates the sound energy in an area, which is too small and poses a greater risk of thermal tissue damage. Based on this information, we would like to realize IC excited drug release in the frequency range mentioned above at the lowest possible pressures, corresponding to a minimal $M I$.

In this study we describe a method to reliably determine the IC pressure threshold employing mono frequent burst sequencies.

\section{Experimental}

\subsection{Measurement Setup}

The IC pressure threshold of the sonosensitive and biocompatible nanoparticles was investigated by utilizing a passive cavitation detection (PCD) test bench (Fig. 1). The PCD setup is partly placed in a water basin, which is filled with degassed, filtered and deionized water. The burst signal is generated via an arbitrary waveform generator (33522B, Agilent), and an RF power amplifier (1140LA, ENI) is used to generate the amplified output signal $u_{\mathrm{S}}(t)$. A matching network is installed to maximize the power transfer to the broadband FUS transducer (H-231, Sonic Concepts ) as well as to transform its electrical impedance at the center frequency of $750 \mathrm{kHz}$ from $27 \Omega$ to $50 \Omega$. A polystyrene cuvette (EXP2.1, $12.5 \mathrm{~mm} \times 12.5 \mathrm{~mm} \times 45 \mathrm{~mm}$, wall thickness $b=1.25 \mathrm{~mm}$,
Carl Roth) mounted in a bracket containing the nanoparticle suspension is placed in the focal volume of the spherically focusing transducer. The sound velocities of water (W) and polystyrene (PS) are $c_{\mathrm{W}}=1500 \mathrm{~m} / \mathrm{s}$ and $c_{\mathrm{PS}}=2340 \mathrm{~m} / \mathrm{s}$, and the acoustic impedances are $Z_{\mathrm{W}} \approx 1.5 \cdot 10^{6} \mathrm{~kg} / \mathrm{m}^{2} \mathrm{~s}$ and $Z_{\mathrm{PS}} \approx 2.35 \cdot 10^{6} \mathrm{~kg} / \mathrm{m}^{2} \mathrm{~s}$. Based on a normal incidence plane wave analysis [10], the amplitude transmission coefficients of the polystyrene cuvette wall at $f=650 \mathrm{kHz} ; 750 \mathrm{kHz}$; $850 \mathrm{kHz}$ become $\tau=(0.93 ; 0.97 ; 0.99)$. Measured values of the standing wave ratio (SWR) of the acoustic pressure are $(1.11 ; 1.1 ; 1.07)$, representing some inhomogeneity of the sound field distribution inside the cuvette. During the FUS exposition the nanoparticles move due to the acoustic radiation pressure, which leads to a certain stirring effect and results in a reasonably uniform overall exposure of the particles. The selected ultrasonic frequencies from $650 \mathrm{kHz}$ to $850 \mathrm{kHz}$ are well focusable due to the concave shape of the transducer surface. This enables the generation of a focused ultrasonic beam, whereby the sound energy is strongly concentrated in a focal volume shaped like an ellipsoid of $2.01 \mathrm{~mm}$ lateral diameter and $14.7 \mathrm{~mm}$ axial size corresponding to the $-6 \mathrm{~dB}$ area. For medical application, the small size of the focal volume is appropriate, as the location of the API release inside the tumorous tissue has to be accurately addressed by the ultrasound.

For measuring the cavitation noise signal of the sonosensitive nanoparticles, we utilize a focusing ultrasonic transducer (V315-SU, Olympus), which is confocally installed in a crossed beam orientation with respect to the FUS beam axis. To reduce unwanted cross-coupled frequency components of the transmitter signal, the received broadband IC noise signal is filtered by a passive highpass filter (2nd order) suppressing the FUS transmitter signal. After filtering, the voltage signal $u_{\mathrm{R}}(t)$ is measured using an digital oscilloscope (DSO7014B, Agilent).

We investigate the CPT at a water temperature of $30{ }^{\circ} \mathrm{C}$, which was kept constant by means of a temperature controller unit. Additionally, a circulating pump was used to achieve a homogeneous temperature distribution inside the water basin. It should be mentioned, that the cavitation pressures inside the cuvette generated by imploding bubbles can not be measured. These bubble implosions are abrasive and would destroy any sensor surface.

\subsection{Sonosensitive Nanoparticles}

The nanoparticles used for these investigations were produced at the Division of Pharmaceutics of the Friedrich-AlexanderUniversity (FAU) Erlangen-Nuremberg. We designed new sonosensitive, biocompatible structures (spheres and capsules) with dimensions from $120 \mathrm{~nm}$ to $250 \mathrm{~nm}$, which also show IC 


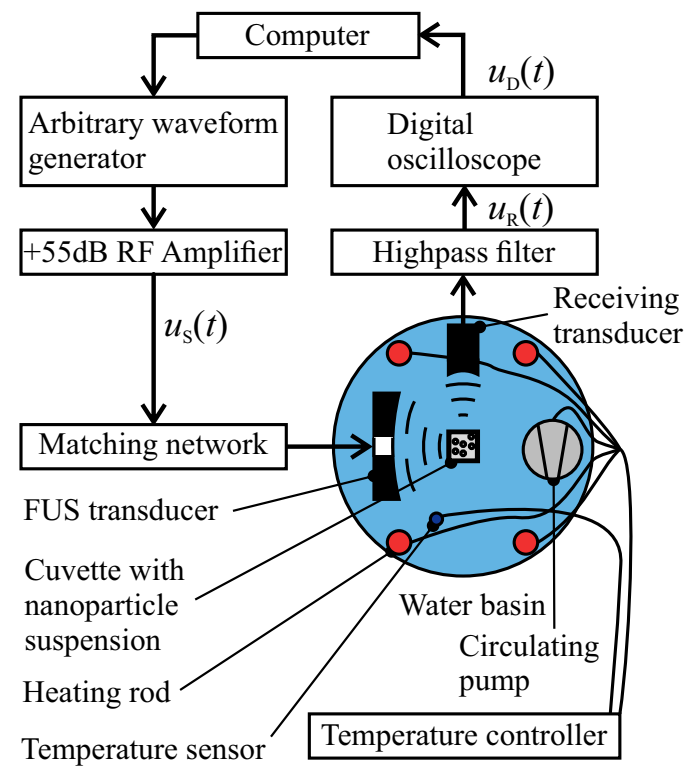

Fig. 1: PCD test bench to investigate the IC pressure threshold.

in a well focusable frequency range. For the production of the nanostructures, an aqueous solution and an organic solution were mixed and then dried by means of a freeze-drying process, resulting in a cake [11]. It is assumed that the nanoparticles have rough surfaces produced at the freeze-drying procedure, which yields to small gas pockets acting as IC bubble nuclei. Furthermore, it is supposed that the API adheres better to a rough surface than to a smooth surface. The PLA-samples were rehydrated with degassed pure water, before the CPT was investigated.

\subsection{Determination of the Cavitation Pressure Threshold}

We determine the CPT in a wide pressure range from $p_{\text {PRFPmin }}=0.2 \mathrm{MPa}$ to $p_{\text {PRFPmax }}=2 \mathrm{MPa}$. The $p_{\text {PRFP }}(m)$ is incremented in $\Delta p_{\mathrm{PRFP}}=0.025 \mathrm{MPa}$ with each measurement $m$ from $m=1$ to $M$ with $M=73$

$$
p_{\mathrm{PRFP}}(m)=p_{\mathrm{PRFPmin}}+(m-1) \cdot \Delta p_{\mathrm{PRFP}} .
$$

A series of sinusoidal burst signals $u_{\mathrm{S}}(t)$ was used to excite IC, according to

$$
u_{\mathrm{S}}(t)=\sin (2 \pi f t) \cdot W(t)
$$

with

$W(t)=\sum_{m=1}^{M} p_{\mathrm{PRFP}}(m) \cdot \operatorname{rect}\left(\frac{t-T_{B} / 2}{T_{B}}-\frac{(m-1) \cdot T_{P}}{T_{B}}\right)$.
The burst length $T_{B}$ was set to $6 \cdot 10^{-4} \mathrm{~s}$ and the period $T_{P}$ lasted $2 \mathrm{~s}$. For each pressure $p_{\mathrm{PRFP}}(m)$, we spectrally analyzed the digitized signal $u_{\mathrm{D}}(t)$ via FFT to evaluate the discrete frequency amplitudes $\mathrm{U}(i)$. Then, we dynamically calculated the voltage spectral density (VSD) $S_{r}$ in the $5.925 \mathrm{MHz}-$ $6.625 \mathrm{MHz}$ domain within a bandwidth $B=0.5 \mathrm{MHz}$ to assess the IC activity of the nanoparticles

$$
S_{r}=\sqrt{\sum_{i=i_{\mathrm{S}}}^{i_{\mathrm{E}}} U(i)^{2}} / \sqrt{B}
$$

with

$$
B=\left(i_{\mathrm{E}}-i_{\mathrm{S}}\right) \cdot \Delta f
$$

and a frequency resolution $\Delta f=1000 \mathrm{~Hz}$. The summation limits $i_{\mathrm{S}}$ and $i_{\mathrm{E}}$ (see Tab. 1) are symmetrically set between the harmonics $f_{\mathrm{h}}$ of the corresponding excitation frequency $f$ and result from

$$
\begin{array}{r}
i_{\mathrm{S}}=f_{\mathrm{S}} / \Delta f=\left(f_{\mathrm{h}}+\left(f_{\mathrm{h}+1}-f_{\mathrm{h}}-B\right) / 2\right) / \Delta f, \\
i_{\mathrm{E}}=f_{\mathrm{E}} / \Delta f=\left(f_{\mathrm{h}+1}-\left(f_{\mathrm{h}+1}-f_{\mathrm{h}}-B\right) / 2\right) / \Delta f .
\end{array}
$$

The spectral evaluation domain from 5.925 MHz-6.625 MHz is based on the filter characteristics of the analog highpass filter.

Tab. 1: Frequency dependent summation limits for the calculation of $S_{r}$.

\begin{tabular}{lccc}
\hline & \multicolumn{3}{c}{$f$ in $\mathrm{kHz}$} \\
& 650 & 750 & 850 \\
\hline$f_{\mathrm{S}}$ in $\mathrm{MHz}$ & 5.925 & 6.125 & 6.125 \\
\hline$f_{\mathrm{E}}$ in $\mathrm{MHz}$ & 6.425 & 6.625 & 6.625 \\
\hline
\end{tabular}

For each frequency $f$ from $650 \mathrm{kHz}$ to $850 \mathrm{kHz}$, we measured $L=15$ nanoparticle samples from $p_{\text {PRFPmin }}$ to $p_{\text {PRFPmax }}$ and determined the mean VSD $\overline{S_{r}}$ corresponding to

$$
\overline{S_{r}\left(f, p_{\mathrm{PRFP}}(m)\right)}=\frac{1}{L} \sum_{j=1}^{L} S_{r j}\left(f, p_{\mathrm{PRFP}}(m)\right) .
$$

Figure 2 shows the mean VSD $\overline{S_{r}}$ at the different frequencies as a function of the PRFP. After that, we calculated the standard deviation $\sigma$ of the VSD $S_{r}$ with

$$
\sigma=\sqrt{\frac{1}{L} \sum_{j=1}^{L}\left|S_{r j}\left(f, p_{\mathrm{PRFP}}(m)\right)-\overline{S_{r}\left(f, p_{\mathrm{PRFP}}(m)\right)}\right|^{2}} .
$$

Finally, we employed a Savitzky-Golay filter (SG) with a polynomial order $p=6$ and an impulse response length $l=17$ for smoothing the standard deviation data. The first maximum of the filtered standard deviation $\sigma_{\max }$ indicates the corresponding CPT. 


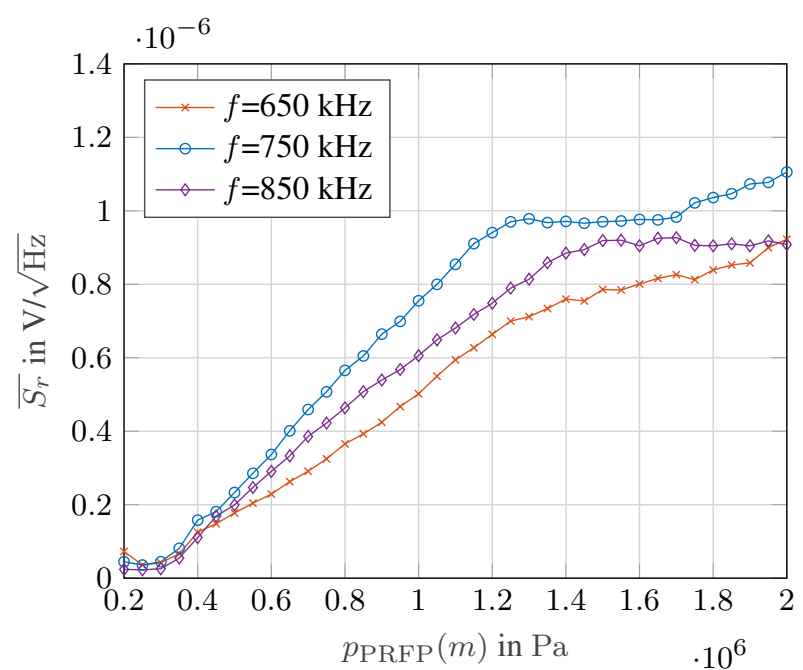

Fig. 2: Progression of the mean VSD $\overline{S_{r}}$ in a pressure range from $0.2 \mathrm{MPa}$ to $2 \mathrm{MPa}$ at frequencies $f$ from $650 \mathrm{kHz}$ to $850 \mathrm{kHz}$.

\section{Results and Conclusion}

Figure 3 depicts the standard deviation (STD) $\sigma$ of the VSD $S_{r}$ from $0.2 \mathrm{MPa}$ to $2 \mathrm{MPa}$ at a frequency $f=650 \mathrm{kHz}$, in which $\sigma_{\max }$ indicates the corresponding CPT $(0.425 \mathrm{MPa})$. The STD increases until the first maximum is reached, then it decreases as the pressure increases. At low pressures, the STD of the VSD is little because IC can not be generated. Close to the CPT, IC is either excited or not resulting in a large STD $\sigma$ of the VSD $S_{r}$. Pressures higher than the CPT lead to a slight decrease of the STD, because IC can be excited reliably. Above a certain pressure, the STD increases again, which is caused by the high non-linearity of IC at large PRFPs. The results of this investigation at different FUS frequencies are listed in Tab. 2. For the used measurement configurations, the lowest CPT of $0.375 \mathrm{MPa}$ as well as the lowest $M I$ of 0.43 are obtained at $f=750 \mathrm{kHz}$, which establishes this frequency as the most suited one for the excitation of IC complying with the FDA regulations for diagnostic ultrasound.

Tab. 2: Results of the CPT investigations at different frequencies.

\begin{tabular}{cccc}
\hline & \multicolumn{3}{c}{$f$ in $\mathrm{kHz}$} \\
& 650 & 750 & 850 \\
\hline$\sigma$ in $\mathrm{nV} / \sqrt{\mathrm{Hz}}$ & 27.3 & 19.6 & 24.9 \\
\hline $\mathrm{CPT}$ in $\mathrm{MPa}$ & 0.425 & 0.375 & 0.45 \\
\hline$M I$ & 0.53 & 0.43 & 0.49 \\
\hline
\end{tabular}

Acknowledgment: The authors gratefully acknowledge the financial support of the Deutsche Forschungsgemeinschaft (DFG) - project no. ER 94/33-1, LE 626/16-1, RU 1656/2-1.

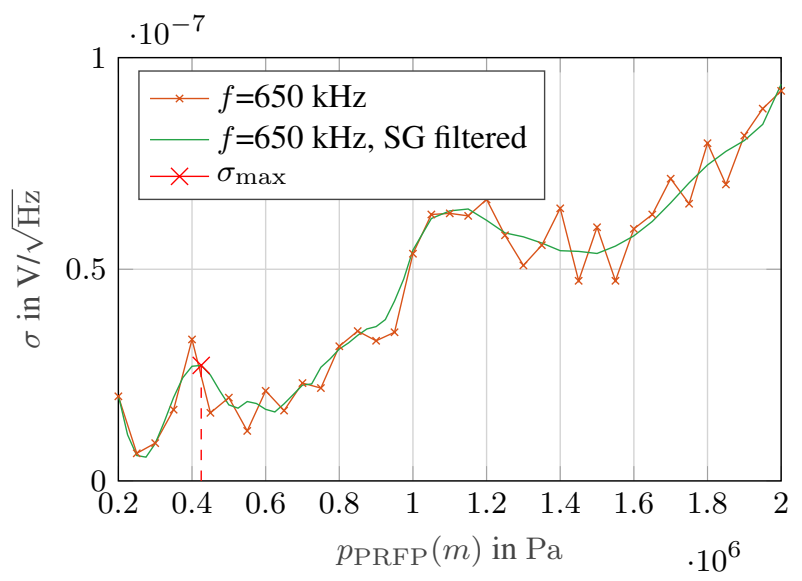

Fig. 3: Unfiltered and filtered standard deviation $\sigma$ of the VSD $S_{r}$ at a frequency $f=650 \mathrm{kHz}$. The first maximum $\sigma_{\max }=$ $0.273 \cdot 10^{-7} \mathrm{~V} / \sqrt{\mathrm{Hz}}$ indicates the CPT at $p_{\mathrm{PRFP}}=0.425 \mathrm{MPa}$.

\section{References}

[1] Yuan $\mathrm{F}$ et al.: Vascular permeability in human tumor xenograft: molecular size dependence and cutoff size. AACR 1995.

[2] Prabhakar $U$ et al.: Challenges and Key Considerations of the Enhanced Permeability and Retention Effect for Nanomedicine Drug Delivery in Oncology. AACR 2013.

[3] Ponce AM et al.: Hyperthermia mediated liposomal drug delivery. Int. J. Hyperthermia 2006.

[4] Meyer DE et al.: Drug targeting using thermally responsive polymers and local hyperthermia. J. Controlled Release 2001.

[5] Tietze $\mathrm{R}$ et al.: Efficient drug-delivery using magnetic nanoparticles-biodistribution and therapeutic effects in tumour bearing rabbits. Nanomed. 2013.

[6] Alexiou $\mathrm{C}$ et al.: Cancer therapy with drug loaded magnetic nanoparticles-magnetic drug targeting. J. Magn. Magn. Mater. 2011.

[7] Pitt WG, Husseini GA, Staples BJ.: Ultrasonic drug delivery a general review. Expert Opin. Drug Deliv. 2004.

[8] Coussios CC, Roy RA.: Applications of Acoustics and Cavitation to Noninvasive Therapy and Drug Delivery. Annu. Rev. Fluid Mech. 2008.

[9] Marketing Clearance of Diagnostic Ultrasound Systems and Transducers: Guidance for Industry and Food and Drug Administration Staff. FDA-2017-D-5372. U.S. Department of Health and Human Services. Rockville, USA. 2019

[10] Kuttruff H.: Physik und Technik des Ultraschalls. S. Hirzel Verlag Stuttgart 1988.

[11] Hiltl PT. et al.: Inertial cavitation of lyophilized and rehydrated nanoparticles of poly(L-lactic acid) at $835 \mathrm{kHz}$ and $1.8 \mathrm{MPa}$ ultrasound. Scientific reports 9(1) 2019. 